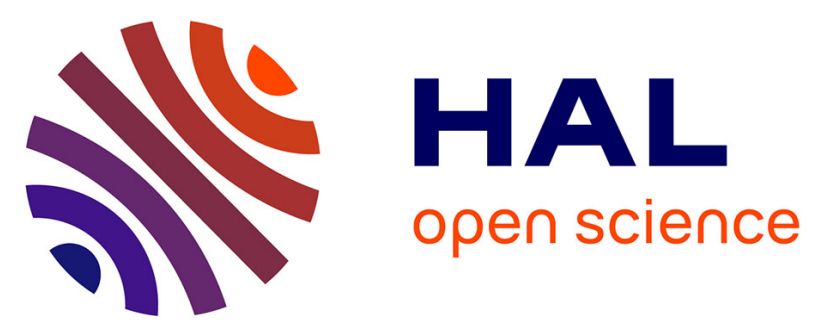

\title{
Angular-resolved energy flux measurements of a dc- and HIPIMS-powered rotating cylindrical magnetron in reactive and non-reactive atmosphere
}

\author{
W P Leroy, S Konstantinidis, S Mahieu, R Snyders, D Depla
}

\section{To cite this version:}

W P Leroy, S Konstantinidis, S Mahieu, R Snyders, D Depla. Angular-resolved energy flux measurements of a dc- and HIPIMS-powered rotating cylindrical magnetron in reactive and non-reactive atmosphere. Journal of Physics D: Applied Physics, 2011, 44 (11), pp.115201. 10.1088/0022$3727 / 44 / 11 / 115201$. hal-00602610

\section{HAL Id: hal-00602610 https://hal.science/hal-00602610}

Submitted on 23 Jun 2011

HAL is a multi-disciplinary open access archive for the deposit and dissemination of scientific research documents, whether they are published or not. The documents may come from teaching and research institutions in France or abroad, or from public or private research centers.
L'archive ouverte pluridisciplinaire HAL, est destinée au dépôt et à la diffusion de documents scientifiques de niveau recherche, publiés ou non, émanant des établissements d'enseignement et de recherche français ou étrangers, des laboratoires publics ou privés. 


\title{
Angular-Resolved Energy Flux Measurements of a DC- and HIPIMS- powered rotating cylindrical magnetron in reactive and non-reactive atmosphere
}

\author{
W.P. Leroy ${ }^{1}$, S. Konstantinidis ${ }^{2}$, S. Mahieu ${ }^{1}$, R. Snyders ${ }^{2,3}$, D. Depla ${ }^{1}$ \\ 1 Research Group DRAFT, Dept. Solid State Sciences, Ghent University, Krijgslaan 281/S1, 9000 Ghent, \\ Belgium \\ 2 Laboratoire de Chimie des Interactions Plasma Surface, CIRMAP, Université de Mons, Place du \\ Parc 20, 7000 Mons, Belgium \\ 3 Materia Nova R\&D center, Avenue Copernic 1, Mons, Belgium
}

A rotating cylindrical magnetron equipped with a titanium target, was sputtered in DC and in HIPIMS mode, both in metallic and in oxide regime. For all sputter modes, the same process conditions and the same average sputtering power of $300 \mathrm{~W}$ were used. An angular-resolved study was performed, 90 degrees around the rotating cylindrical magnetron, which obtained the total energy flux arriving at the substrate. Furthermore, the energy flux per adparticle was calculated by measuring the deposition rate for all sputter modes and regimes. There is only a small difference in total arriving energy flux between the DC-mode and the HIPIMS mode. A maximum arriving energy flux of ca. $0.26 \mathrm{~mW} / \mathrm{cm}^{2}$ was measured, when normalized to the sputtering power. Concerning the deposition rate, up to a $75 \%$ decrease was found from DC to HIPIMS mode. Furthermore, the emission and the transport of the particles have a similar angular profile for all sputter modes. Among the HIPIMS modes, a decrease in deposition rate was measured with increasing pulse length. Therefore, the energy which arrives per adparticle is the highest for the HIPIMS modes. A difference in the angular shape of the energy per arriving adparticle is noticed between the DC and the HIPIMS modes. The DC mode has a maximum arriving energy per adparticle around 50 degrees, while this is at 60 degrees for the HIPIMS mode.

\section{Introduction}

Nowadays, the use of functional coatings is widely spread and numerous applications and tools rely on the properties and stability of the functional coating. To apply these functional thin films, a wide variety of deposition techniques can be used. For many applications, physical vapor deposition (PVD) has prevailed as the method of choice. Moreover, magnetron sputtering can be considered as the most industrially favored PVD technique, due to its high-throughput production of high quality thin films.

In the 1980 s, a rotating cylindrical magnetron was developed[1,2] to improve target utilization. As magnetron sputtering has many industrially relevant advantages, the erosion profile due to the presence of a stationary racetrack often limits the target consumption and increases its cost of ownership[3]. A rotating cylindrical magnetron has a superior target utilization (up to 70\% or more), as compared to planar magnetrons[2]. Furthermore, higher power levels (and hence higher deposition rates) are possible due to its better target cooling. Therefore, these magnetrons are now the workhorse of many industries that are doing large-area and in-line coatings. Products of these industries include flat panel displays, low-emissivity and solar control windows and thin film photovoltaics. Furthermore, rotating cylindrical magnetrons have proven their worth in research, as to exhibit certain fundamental effects in the magnetron sputtering process (e.g. the influence of redeposition on the behavior in reactive sputtering), which would not have been possible for a planar magnetron[4-6].

Recently, there has been a growing interest in the process of high power impulse magnetron sputtering (HIPIMS)[7-10], both among academics as industrials. HIPIMS discharges generate a dense plasma, allowing a high degree of ionization of the sputtered (film-forming) atoms. With this PVD technique, smooth coatings are being deposited with a high density, even on complex-shaped 
substrates[7]. However, some drawbacks to the technique include a lower deposition rate as compared to conventional DC sputtering, a complex and not well-understood behavior in reactive atmosphere, and technological complications for upscaling[10]. Still, HIPIMS can be regarded as a very interesting and promising technique for industrial applications.

The combination of these two industrially favored techniques, namely HIPIMS using a rotating cylindrical magnetron, seems like a winning combination for different industries. Recently, the first successful combination was reported[11]. Key to the use of a certain PVD technique in a process is its energy flux towards the substrate, as these substrates are often sensitive to high temperature (e.g. flexible solar panels). Furthermore, the energy flux towards the substrate plays a vital role in the growth behavior of the thin film[12,13]. For the latter, the energy per arriving adparticle is of high importance. Therefore, the arriving adparticles (or the deposition rate) will be compared for both DC and HIPIMS mode. Additionally, both operating modes will be tested in oxide regime ('full poisoning'), i.e. when the target surface is completely oxidized[14, 15]. The aim of the present work is to give more detailed analysis of the behavior of a rotating cylindrical magnetron, when applying HIPIMS.

\section{Experimental}

All experiments were performed in a stainless-steel vacuum system of which a sketch is shown in figure 1 . The chamber was evacuated using a turbomolecular pump to a base pressure of $10^{-4} \mathrm{~Pa}\left(10^{-6}\right.$ mbar). Before the experiments, the pumping speed was set to $48 \mathrm{~L} / \mathrm{s}$ using a valve. The working pressure of Ar for the experiments was kept constant at $0.7 \mathrm{~Pa}$. The oxygen flow was regulated using a mass flow controller (Aera) and in oxide regime it was set at $4 \mathrm{sccm}$ (standard cubic centimeter per minute) yielding a total pressure of ca. $0.8 \mathrm{~Pa}$. In the oxide regime, the target surface was fully poisoned, as was evidenced by the DC target voltage and the quartz microbalance measurements.

A next generation of the small-scaled, rotating cylindrical magnetron as originally described in previous work[16] has been used. The most important change to the setup was the use of a different magnet system, which produces a higher maximal, radial magnetic field strength of $1000 \mathrm{G}$. The magnet configuration can be rotated to any fixed position within the target, which enables angularresolved measurements (see figure 1). The area of the racetrack was ca. $10 \mathrm{~cm}^{2}$. The Ti target is a tube with a length of $185 \mathrm{~mm}$ and an outer diameter of $48 \mathrm{~mm}$. The target rotated at a speed of $20 \mathrm{rpm}$.

The HIPIMS power supply used in this work[17] allows the generation of pulses whose length was varied between $5 \mu \mathrm{s}, 15 \mu \mathrm{s}$ and $20 \mu \mathrm{s}$ with a repetition frequency of $5700 \mathrm{~Hz}, 700 \mathrm{~Hz}$ and $500 \mathrm{~Hz}$ respectively. These are relatively short HIPIMS pulses compared to other groups $[8,10,18]$. For all HIPIMS experiments, the applied voltage was set around $600 \mathrm{~V}$, resulting in a peak current of about $75-100 \mathrm{~A}$ and obtaining a constant average power of 300W $( \pm 5 \%)$. The current and voltage waveforms were recorded at the magnetron side. For the DC measurements, a ENI RPG 50 power supply was used to deliver constant current (ca. 0.9 A) to match the average power in the HIPIMS discharge. Hence, the peak power obtained with the HIPIMS power supply is typically two orders of magnitude larger than the one reached during the DC experiments. However, to have the best description of the processes, all measurements will be normalized to the average power. This will allow for a well-founded comparison.

A passive thermal probe measured the total energy flux towards the substrate[19, 20]. Deposition rates were measured with a quartz crystal microbalance (Maxteck, Inc. Thickness monitor model TM350 ) facing the target, at the same distance as the thermal probe $(12 \mathrm{~cm})$. Both probes were grounded during the measurements.

\section{Results and Discussion}

\section{HIPIMS pulse}


In previous work[11], it was shown that a stable operation of a rotating cylindrical magnetron during HIPIMS sputtering can be obtained. Based on the I-V characteristics (see figure 2) from the HIPIMS discharges, this can be confirmed for both metallic as well as oxide mode.

When comparing the shape of the voltage-current pulses for the two sputter regimes (metallic and oxide), a difference in the current behavior can be distinguished. For the metallic regime, there is a linear increase of the current with time, as long as the voltage pulse is running. This is expected[21], and as shown by Lundin et al.[22], the discharge current would drop back to a lower plateau value (around the DC value), if the pulse duration would be extended (i.e. more than $100 \mu$ s).

When examining the current pulse in oxide regime, there is at first a slower, linear increase of the current with pulse duration. However, after a few microseconds the current starts to increase more rapidly and finally overtakes the one of the metallic regime. This different shape of the current curve, namely a sudden, sharp increase and "bent" form of the current pulse is typical for the oxide regime and was noticed throughout all oxide measurements.

When the target is operated in oxide regime, the surface completely consists of oxide material. This oxide layer is not removed within one HIPIMS pulse. As a rough estimate, it can be considered that during one pulse about $1.5 \times 10^{15}$ ions arrive at the target surface, if the discharge current pulse is considered to be supplied solely by singly charged ions. In this estimate, the contribution of negatively charged species (e.g. the secondary electrons, negative oxygen ions) to the current is neglected and hence the calculated amount of arriving ions is an upper limit. Furthermore, it is assumed that the target only gets sputtered by Ar ions. This is a good approximation, as the metal selfsputtering is negligible for short pulse lengths[21]. With the area of the racetrack region being $10 \mathrm{~cm}^{2}$, the flux of ions per pulse towards the target surface is $1.5 \times 10^{14}$ ions $/ \mathrm{cm}^{2}$. Considering that the sputter yield of titanium oxide is 10 to 20 times lower than the one of metallic titanium, which is 0.67[23] for these conditions, it is clear that no complete monolayer of the target surface (around $10^{15}$ atoms $/ \mathrm{cm}^{2}$, [24]) gets sputtered off during one HIPIMS pulse. Therefore, the target surface will remain oxidized during the pulse and hence this cannot explain the sharp increase of the current. Some different mechanisms must kick in, which should originate from the plasma chemistry. The plasma has a different composition due to the added oxygen. These oxygen molecules are also ionized and produce some oxygen atoms by dissociation, which are in turn ionized. Time-resolved OESmeasurements support the notion of increased oxygen dissociation during the high-power pulse [25]. This concentration of atomic oxygen increases with a certain delay from the start of the pulse, as compared to the argon concentration. Hence these oxygen-related processes start contributing to the current with a certain delay and then make the current increase more than linearly. The same behavior was observed and discussed by Benzeggouta et al.[26] for the efficiency of pre-ionization in the case of HIPIMS-sputtering of a ruthenium target in an $\mathrm{Ar} / \mathrm{O}_{2}$ atmosphere.

\section{Deposition Rates}

In this section, the advantage of the rotating cylindrical magnetron is that the magnets can be turned within the cathode. This allows for a complete angular description of the plasma parameters. Hence, deposition rate measurements were carried out by systematically moving the cathode magnets by steps of $10^{\circ}$. Figure $3(\mathrm{a})$ shows that the deposition rate drops when the magnets (and hence the racetrack) are rotated away from the quartz balance. For DC, this shape can be perfectly simulated using the test-particle Monte Carlo code SIMTRA[27]. Furthermore, a more than 75\% decrease can be observed for the deposition rate in HIPIMS mode (with a $20 \mu$ s long pulse), compared to DC mode.

The ratio of the deposition rate as a function of angle is shown in figure $3(\mathrm{~b})$, where the deposition rate for each sputter mode is normalized to the deposition rate of the DC mode. This allows for a good comparison of the angular distribution for each of the sputter modes.

Among the different HIPIMS modes, there is a decrease in deposition rate with the increase of the pulse length. This phenomenon has been discussed by e.g. Konstantinidis et al.[21], and is often attributed to the self-sputtering regime. With longer pulse durations, a higher density of ionized metal particles is obtained and self-sputtering becomes important to dominant in the process. However, 
other processes will contribute to this phenomenon: the depletion of the local gas density ("gas rarefaction")[28, 29], a different emission profile, different particle transport throughout the gas phase $[30,31]$. The latter two processes can cause a different material distribution throughout the deposition chamber, hence yielding a locally changed deposition rate.

However, Figure 3(b) clearly demonstrates that the shape of the angular distribution is identical for all sputter modes. This inclines that the emission profile and the transport of the particles is the same for all sputter modes. Horwat and Anders noticed the same when making an angular resolved study around a planar magnetron with a $\mathrm{Cu}$ HIPIMS discharge [32]. This behavior would imply that the anomalous transport evidenced by Lundin et al.[30], which enhances the sideways ejection of metal ions in the case of a circular magnetron geometry wouldn't apply for a rectangular magnet configuration, as used in the present study. Or that sideway emission is in a different direction than measured here. One could also assume that another effect counterbalances the phenomenon discussed by Lundin et al. in such a way that the HIPIMS and DC profiles are identical for our working conditions.

Furthermore, the normalized ratios of the deposition rate are shown in the legend of figure 3(b). Compared to DC, the deposition rate drops significantly (to half) when using the HIPIMS $5 \mu$ s mode. For longer pulse times, there seems to be a stabilization and the decrease in deposition rate is less

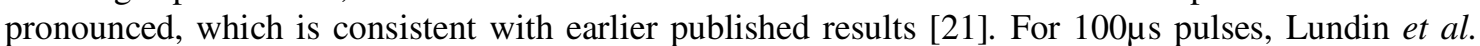
[33] observed a decrease to $20 \%$ of the DC-deposition rate. This observation agrees with the trend noticed here.

When the cathode is sputtered in oxide regime, the deposition rate drops significantly for both modes (DC and HIPIMS). This phenomenon is already well-known[14, 15], but the deposition rate for the HIPIMS oxide regime is again lower than for the DC oxide regime, as can be observed in figure 3(a). The deposition rate in DC-oxide regime drops to $6 \%$ of the normal DC deposition rate. For the HIPIMS-oxide regime this drop is as expected even bigger, namely to $3 \%$. Comparing the oxide regimes to their respective metallic regimes, i.e. the HIPIMS $5 \mu$ s oxide regime to the HIPIMS $5 \mu \mathrm{s}$ regime, the drop in deposition rate is equal (for HIPIMS: 0.03/0.47=0.06). This indicates that the change in sputter behavior (i.e. sputter yield and electron emission coefficient) is the same for both sputtering modes (DC and HIPIMS), when going from a metallic target to an oxidized target.

\section{Energy Flux}

The energy flux towards the substrate plays an important role in the deposition process. It can influence the growth behavior of the thin film, or damage temperature-sensitive substrates. HIPIMS has been proposed as an ideal deposition technique to avoid temperature damage to the substrate[18]. Therefore, the total energy flux for the rotating cylindrical magnetron was measured for the different deposition modes.

Figure 4(a) shows the angular dependence of the total energy flux for the different operating modes. The values for the arriving energy flux $\left(\mathrm{E}_{\mathrm{flux}}\right)$ have been normalized to the average power $\left(\mathrm{P}_{\mathrm{av}}\right)$ of the used deposition mode. Overall, the angular dependence is similar for all operating modes, with the highest arriving total energy flux for the DC-mode. All sputter modes exhibit a maximum energy flux around 40 degrees. The difference in total arriving energy flux is however small between the DC-


operation could therefore be considered as the limit of the HIPIMS mode for which the pulse duration is infinitely short $(0 \mu \mathrm{s})$ and the frequency infinitely large.

For the different HIPIMS modes, there is a small decrease in total energy flux with increasing pulse length. Lundin et al.[33] and West et al.[18] also reported on the arriving energy flux during HIPIMS. However, different HIPIMS parameters were used, which makes it hard to compare. To study the several measurements, the total energy flux of the HIPIMS mode is normalized to the one of the DC mode. These ratios can be seen in figure 4(b), as plotted as a function of their pulse length. Figure 4(b) shows a decrease in this ratio with increasing pulse lengths. Furthermore, the values reported in literature confirm our findings, if the observed trend is extrapolated to a $100 \mu \mathrm{s}$ pulse 
length (dashed line). Overall, the total energy flux is lower for the HIPIMS modes than for the DC mode. However, for short pulse lengths, this difference becomes quite small.

This behavior could originate from the total amount of particles (Ar fast neutrals and ions, sputtered metal neutrals and ions, electrons, ...) that reach the probe. Indeed, it has been shown that both the ionization and deposition rates depend on the pulse duration (at constant discharge voltage)[21]. The larger the pulse duration, the larger is the ionization rate but the lower is the deposition rate. Hence the energy must be calculated with respect to the number of incoming adparticles.

\section{Energy flux per adparticle}

Not only the total energy flux arriving at the substrate plays a vital role, but the energy per arriving adparticle plays a crucial role in the growth behavior of the thin film[12, 13]. It can be either desirable (e.g. interface engineering[34]) or detrimental (e.g. high-energy negative oxygen ions[35]), depending on the material and the property wanted. Therefore, figure 5 shows the energy flux per arriving Ti adparticle for the different sputter modes, with figure 5(b) showing an enlarged view for the metallic regimes.

Considering the overall values for the energy flux per adparticle, the DC mode has the lowest values. This is followed by the HIPIMS mode with $5 \mu$ s pulse length, which already exhibits 1.5 times more energy per adparticle than the DC mode. For the longer pulse lengths, even higher amounts are observed, raising to about 2.8 times and 3 times the energy of the DC mode, for the $15 \mu \mathrm{s}$ and $20 \mu \mathrm{s}$ pulse length respectively. The two oxide regimes clearly have the highest arriving energy flux per arriving adparticle. Compared to their metallic regime, the DC oxide regime exhibits a 12 times higher energy flux per arriving adparticle, while for the HIPIMS oxide regime this is almost 19 times higher. The dramatic increase of the overall energy per adparticle when working in the oxide regime could be attributed to the significant amount of negative ions such as $\mathrm{O}^{-}$reaching the probe surface. These ions have been detected in a DC magnetron discharges [35, 36] and in HIPIMS plasmas[37]. Negative ions are sputtered from the oxidized target and accelerated by the cathode sheath. Hence they may reach the probe with very high energies of the order of $300 \mathrm{eV}$ during our DC magnetron sputtering experiments and $600 \mathrm{eV}$ during HIPIMS.

The angular shape of this energy flux per adparticle looks quite puzzling. The overall trend is a rise in the energy flux per adparticle with higher angles. However, a decrease occurs for all sputter modes, albeit at different angles. For the DC mode this decrease happens around 50 degrees, while the HIPIMS $20 \mu \mathrm{s}$ (and roughly speaking the $5 \mu$ s and $15 \mu$ s too) have their maximum at 60 degrees.

Mahieu et al.[12] measured and calculated the different contributions to the total energy flux per incoming adparticle for reactive DC magnetron sputtering of TiN. It is evidenced that the main contribution (depending on the working conditions used) originates from the ion flux, and to a lesser extent from the electrons and the sputtered particles. It is therefore obvious to observe that the HIPIMS discharge, characterized by an increased ionization rate, exhibits i) an increased energy flux per adparticles as compared to DC and ii) that during HIPIMS the energy flux increases when increasing the pulse duration. The ionization rate of the sputtered metal vapor was found to increase as the pulse duration was increased (at constant discharge voltage) [21].

As concluded from the first section, the angular emission profile of the sputtered particles is similar for DC and HIPIMS mode. Therefore the difference in the angular shape of the total energy per adparticle probably arises from a changed electron and (positive and negative) ion distribution. More research will be conducted to compare these distributions for DC and HIPIMS mode on a rotating cylindrical magnetron by e.g. Langmuir probe, optical absorption spectroscopy and mass spectrometry. There exists some data in literature which already indicate a difference between the two modes of operation. Lundin et al.[30] observed a radial acceleration of the ions over the target surface, which they attribute to an anomalous electron transport. However, the data reported by Lundin et al. were acquired for a circular target (and racetrack), not for a rectangular one. It is known from the literature that the rectangular target geometry exhibits some discrepancies regarding the motion of the electrons (e.g. the cross-corner effect). Furthermore, Bohlmark et al. [38] showed a 
two-stage deformation of the magnetic field during the HIPIMS pulse. This could an effect which causes a change in the transport of the charged particles, hence causing a change in the angular shape of the arriving energy flux per adparticle. These example highlight the need for more comparative experiments, in which the option for angular distributions using the rotating cylindrical magnetron will definitely prove its worth.

\section{Conclusions}

A rotating cylindrical magnetron is successfully operated in HIPIMS mode, both in metallic as in oxide regime. A difference in the shape of the current pulse is ascribed to a different plasma chemistry when sputtering in oxide regime, namely a delayed dissociation-ionization of the oxygen molecules. This gives rise to a faster-than-linear increase of the current.

A comparison is presented of the energy flux from a rotating cylindrical magnetron for sputtering in DC mode and in HIPIMS mode. This is done for both the metallic as the oxide regime in function of angle around the cylindrical target. The total energy flux is quantitatively compared by determining the adparticle flux (from the deposition rate) as a function of angle, for each mode.

The deposition rate of the HIPIMS mode is lower than for the DC mode, and it decreases with increasing pulse length. The decrease in deposition rate stabilizes for higher pulse lengths. Furthermore, the angular emission profile and the transport of the particles is the same for all sputter modes.

The angular dependence of the total energy flux is similar for all operating modes, with the highest arriving total energy flux for the DC-mode. The difference in total arriving energy flux is however small between the DC-mode and the HIPIMS mode, especially for the short pulse durations (e.g. $5 \mu \mathrm{s})$. The decrease in total energy flux with increasing pulse length confirms previous work by other groups.

The energy which arrives per adparticle is the highest for both oxide regimes. Among the metallic regimes, the HIPIMS modes exhibit a much higher energy per adparticle, with the highest values for the longer pulse lengths. This effect would originate from the increased ionization rate of the sputtered material in HIPIMS as the pulse duration is increased. Furthermore, a difference in the angular shape of the energy per arriving adparticle is noticed between the DC and the HIPIMS modes. The DC mode has a maximum arriving energy per adparticle around 50 degrees, while this is at 60 degrees for the HIPIMS mode.

\section{Acknowledgements}

This work was partially funded by the Institute for the Promotion of Innovation through Science and Technology in Flanders (IWT-Vlaanderen), under SBO Project No. 60030. Furthermore, the WOG: "Structural and chemical characterization of materials at the micro- and nanoscale" is acknowledged for its support. S.M. is a Postdoctoral Fellow of the Research Foundation-Flanders (FWO) and S.K. is Postdoctoral Fellow of the National Funds for Scientific Research (FNRS). S.K. and R.S. thank the Interuniversity Attraction Poles Program of the Belgian Science Policy (Project "PSI: Fundamentals of Plasma Surface Interactions") for financial support.

\section{References}

[1] McKelvey H E 1982 Magnetron Cathode Sputtering Apparatus, patent (US, 4,356,073)

[2] Wright M and Beardow T 1986 J. Vac. Sci. Technol. A-Vac. Surf. Films 4 388-92

[3] De Bosscher W and Lievens H 1999 Thin Solid Films 351 15-20 
[4] Li X Y, Depla D, Leroy W P, Haemers J and De Gryse R 2008 Journal of Physics D-Applied Physics 41035203

[5] Mahieu S, Leroy W P, Depla D, Schreiber S and Moller W 2008 Appl. Phys. Lett. 93061501

[6] Depla D, Li X Y, Mahieu S, Van Aeken K, Leroy W P, Haemers J, De Gryse R and Bogaerts A 2010 J. Appl. Phys. 1079

[7] Kouznetsov V, Macak K, Schneider J M, Helmersson U and Petrov I 1999 Surface \& Coatings Technology 122 290-3

[8] Helmersson U, Lattemann M, Bohlmark J, Ehiasarian A P and Gudmundsson J T 2006 Thin Solid Films 513 1-24

[9] Hovsepian P E, Ehiasarian A P, Deeming A and Schimpf C 2008 Vacuum 82 1312-7

[10] Sarakinos K, Alami J and Konstantinidis S 2010 Surface and Coatings Technology 204 166184

[11] Leroy W P, Mahieu S, Depla D and Ehiasarian A P 2010 J. Vac. Sci. Technol. A 28 108-11

[12] Mahieu S and Depla D 2009 Journal of Physics D-Applied Physics 42053002

[13] Kersten H, Deutsch H, Steffen H, Kroesen G M W and Hippler R 2001 Vacuum 63 385-431

[14] Depla D and De Gryse R 2004 Surface \& Coatings Technology 183 184-9

[15] Berg S, Nyberg T and Kubart T 2008 Reactive Sputter Deposition, ed D Depla and S Mahieu (Berlin: Springer-Verlag)

[16] Depla D, Haemers J, Buyle G and De Gryse R 2006 J. Vac. Sci. Technol. A 24 934-8

[17] Ganciu M, Konstantinidis S, Paint Y, Dauchot J P, Hecq M, de Poucques L, Vasina P, Mesko M, Imbert J C, Bretagne J and Touzeau M 2005 J. Optoelectron. Adv. Mater. 7 2481-4

[18] West G, Kelly P, Barker P, Mishra A and Bradley J 2009 Plasma Process. Polym. 6 S543-S7

[19] Stahl M, Trottenberg T and Kersten H 2010 Rev. Sci. Instrum. 814

[20] Wolter M, Stahl M and Kersten H 2009 Plasma Process. Polym. 6 S626-S30

[21] Konstantinidis S, Dauchot J P, Ganciu M, Ricard A and Hecq M 2006 J. Appl. Phys. 995

[22] Lundin D, Brenning N, Jadernas D, Larsson P, Wallin E, Lattemann M, Raadu M A and Helmersson U 2009 Plasma Sources Sci. Technol. 186

[23] Saraiva M, Georgieva V, Mahieu S, Van Aeken K, Bogaerts A and Depla D 2010 J. Appl. Phys. 10710

[24] Ohring M 2002 Materials Science of Thin Films (San Diego, London: Academic Press)

[25] Konstantinidis S, Gaboriau F, Gaillard M, Hecq M and Ricard A 2008 Reactive Sputter Deposition, ed D Depla and S Mahieu: Springer) p 301

[26] Benzeggouta D, Hugon M C, Bretagne J and Ganciu M 2009 Plasma Sources Sci. Technol. 189

[27] Van Aeken K, Mahieu S and Depla D 2008 Journal of Physics D-Applied Physics 416

[28] Rossnagel S M 1988 J. Vac. Sci. Technol. A-Vac. Surf. Films 6 19-24

[29] Anders A and Yushkov G Y 2009 J. Appl. Phys. 1056

[30] Lundin D, Larsson P, Wallin E, Lattemann M, Brenning N and Helmersson U 2008 Plasma Sources Sci. Technol. 176

[31] Konstantinidis S, Dauchot J P, Ganciu M and Hecq M 2006 Appl. Phys. Lett. 883

[32] Horwat D and Anders A 2008 Journal of Physics D-Applied Physics 416

[33] Lundin D, Stahl M, Kersten H and Helmersson U 2009 Journal of Physics D-Applied Physics 427

[34] Ehiasarian A P, Wen J G and Petrov I 2007 J. Appl. Phys. 101054301

[35] Mahieu S, Leroy W P, Van Aeken K and Depla D 2009 J. Appl. Phys. 106

[36] Mraz S and Schneider J M 2006 J. Appl. Phys. 1006

[37] Sarakinos K, Music D, Mraz S, Baben M T, Jiang K, Nahif F, Braun A, Zilkens C, Konstantinidis S, Renaux F, Cossement D, Munnik F and Schneider J M 2010 J. Appl. Phys. 108014904

[38] Bohlmark J, Helmersson U, VanZeeland M, Axnas I, Alami J and Brenning N 2004 Plasma Sources Sci. Technol. 13 654-61 


\section{List of Figures}

Figure 1 (color online): Sketch of the experimental setup. The magnet array (and hence the racetrack) can be positioned at any angle $\alpha$ to the used process monitor. The tubular Ti target rotates around the stationary magnet array with a cerain speed.

Figure 2 (color online): Current and voltage waveforms during sputtering of a rotating cylindrical magnetron in HIPIMS mode ( $5 \mu$ s pulse width), in metallic and oxide regime.

Figure 3 (color online): (a) The deposition rate $\left(\mathrm{R}_{\mathrm{d}}\right)$ as measured by a quartz microbalance, as a function of angle around the rotating cylindrical magnetron, for different sputter modes. (b) The deposition rates relative to the deposition rate of the DC sputter mode, as a function of angle around the rotating cylindrical magnetron.

Figure 4 (color online): (a) Polar plot of the total arriving energy flux for the different sputter modes, as a function of angle of rotation around the rotating cylindrical magnetron, normalized to the average sputtering power. (b) Ratio of the total energy flux for the HIPIMS mode to the DC mode, plotted as a function of pulse length. A comparison to literature values (West et al.[18] and Lundin et al.[33]) is given. The dashed line is a guide to the eye.

Figure 5 (color online): Energy flux per arriving adparticle for the different sputer modes. (b) is a magnification of the region boxed in (a). 


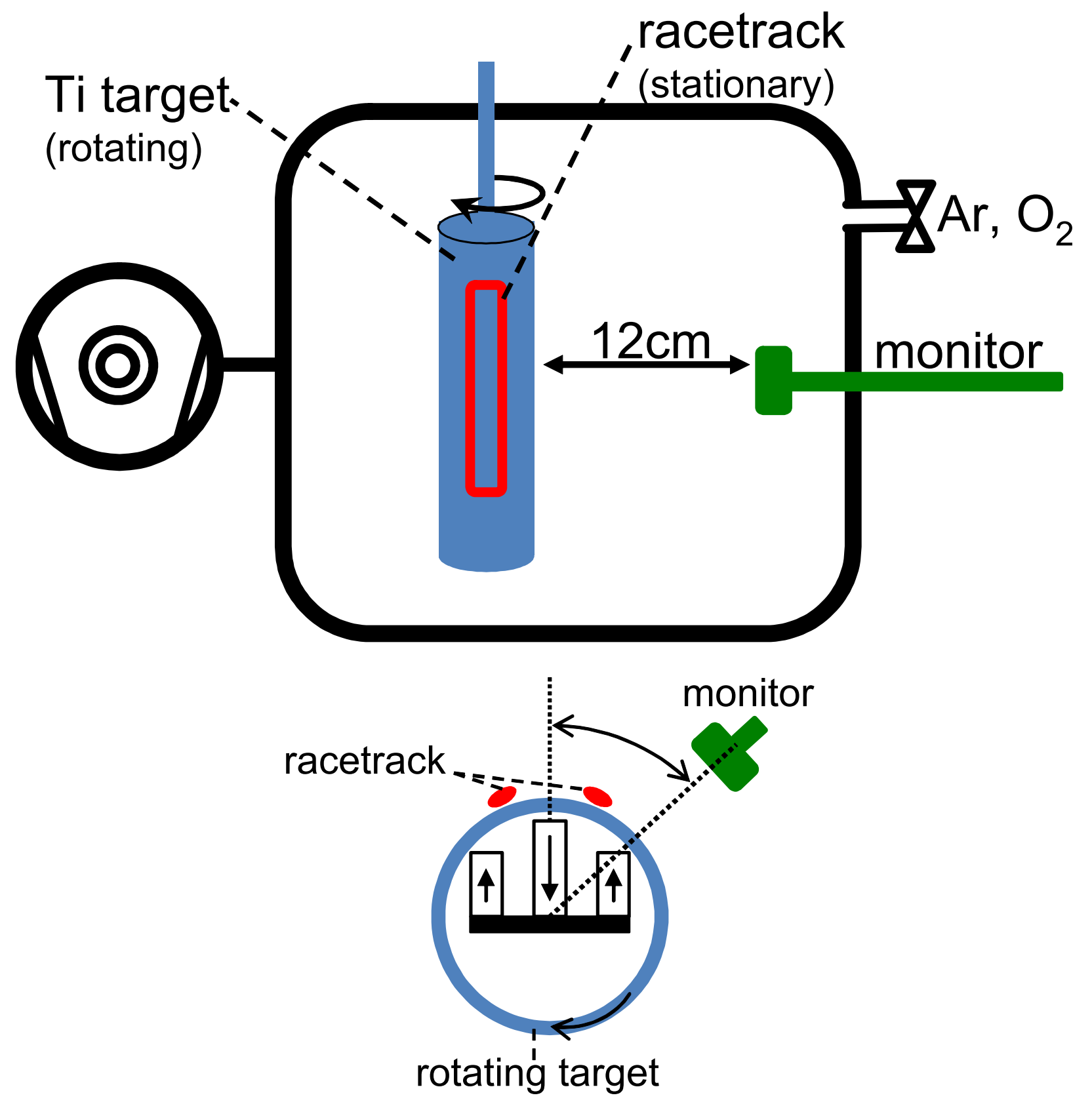

Figure 1 (WouterLeroy_Revision_Figure1.eps) 


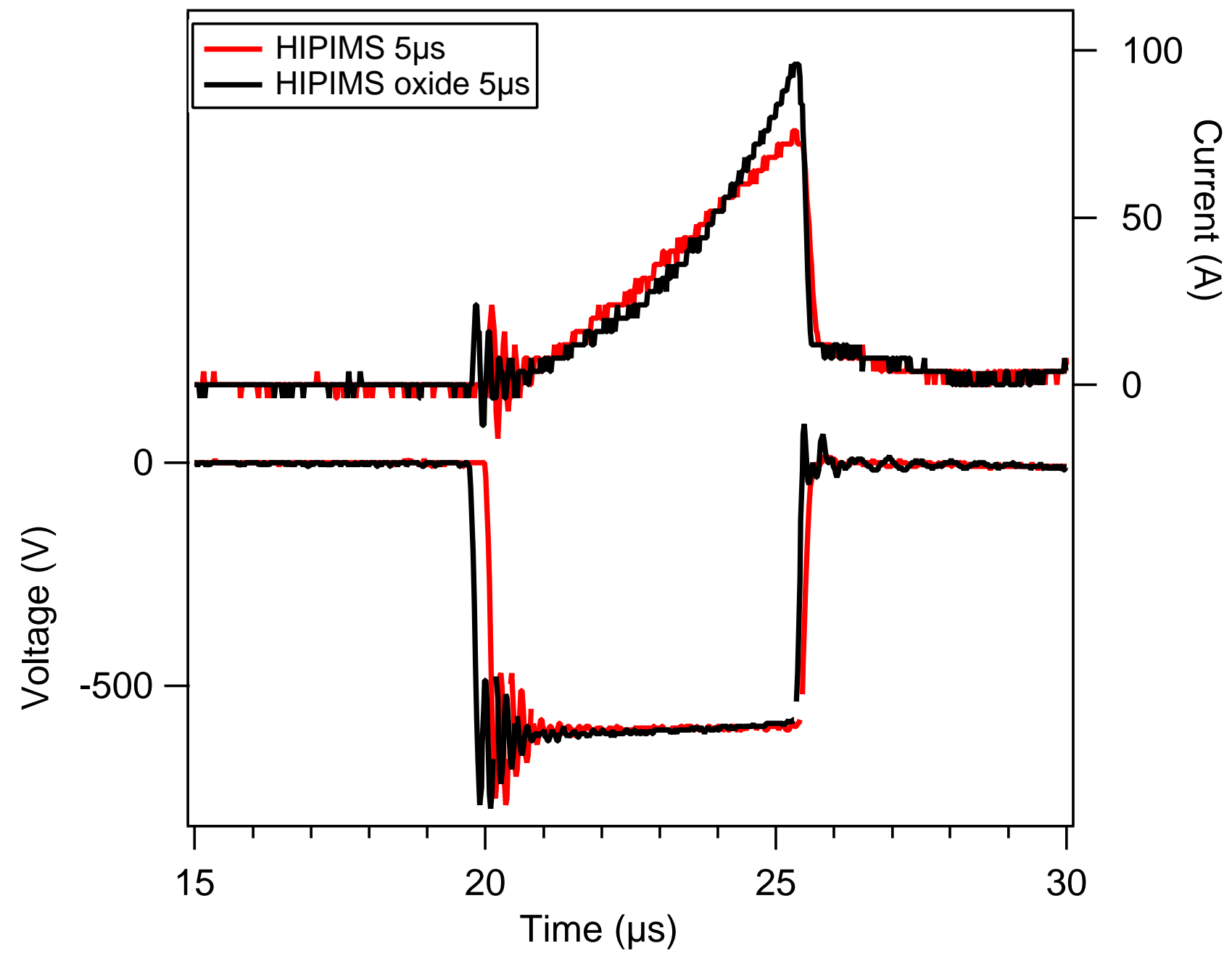

Figure 2 (WouterLeroy_Revision_Figure2.eps) 

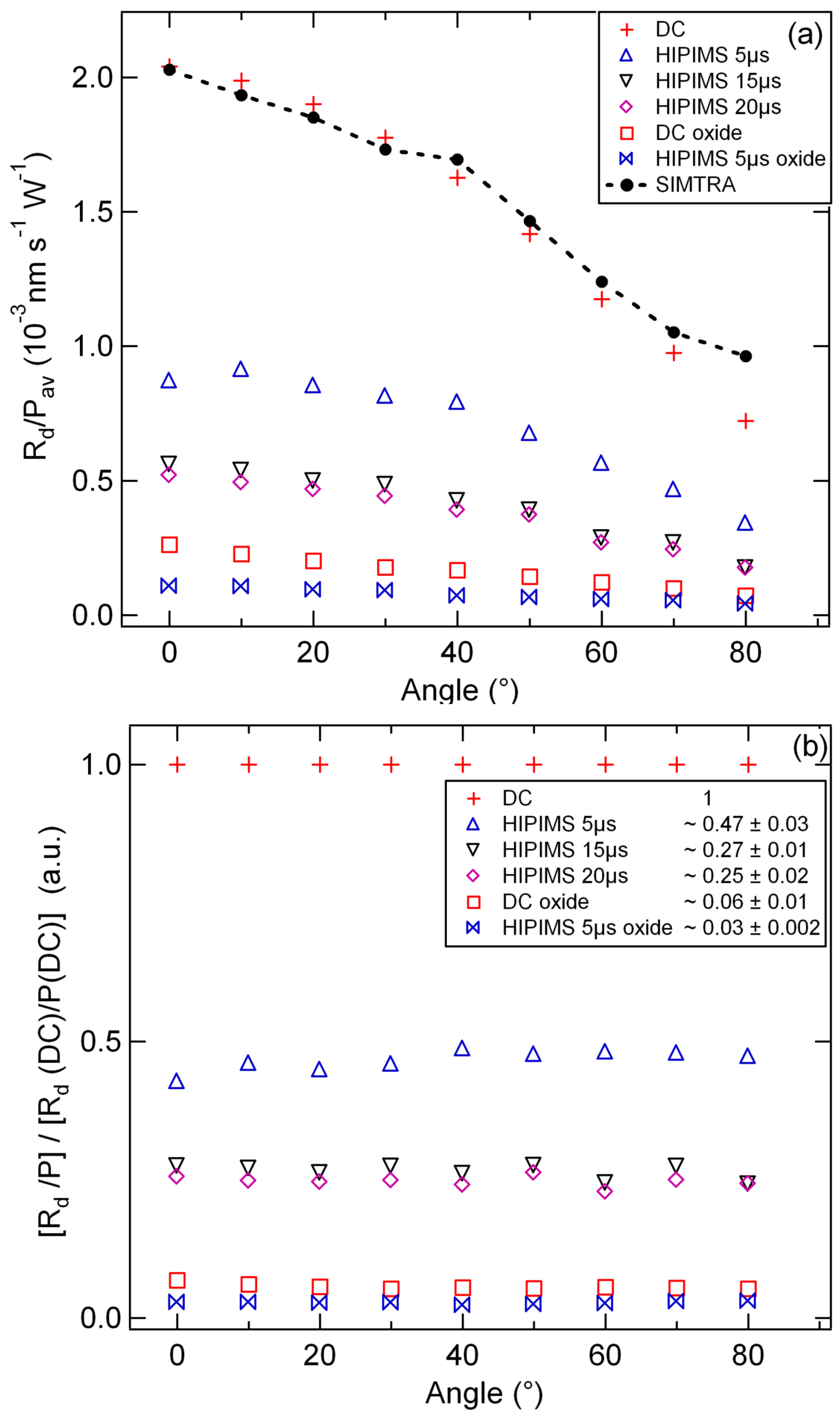

Figure 3 (WouterLeroy_Revision_Figure3.eps) 



Figure 4 (WouterLeroy_Revision_Figure4.eps) 
(a)

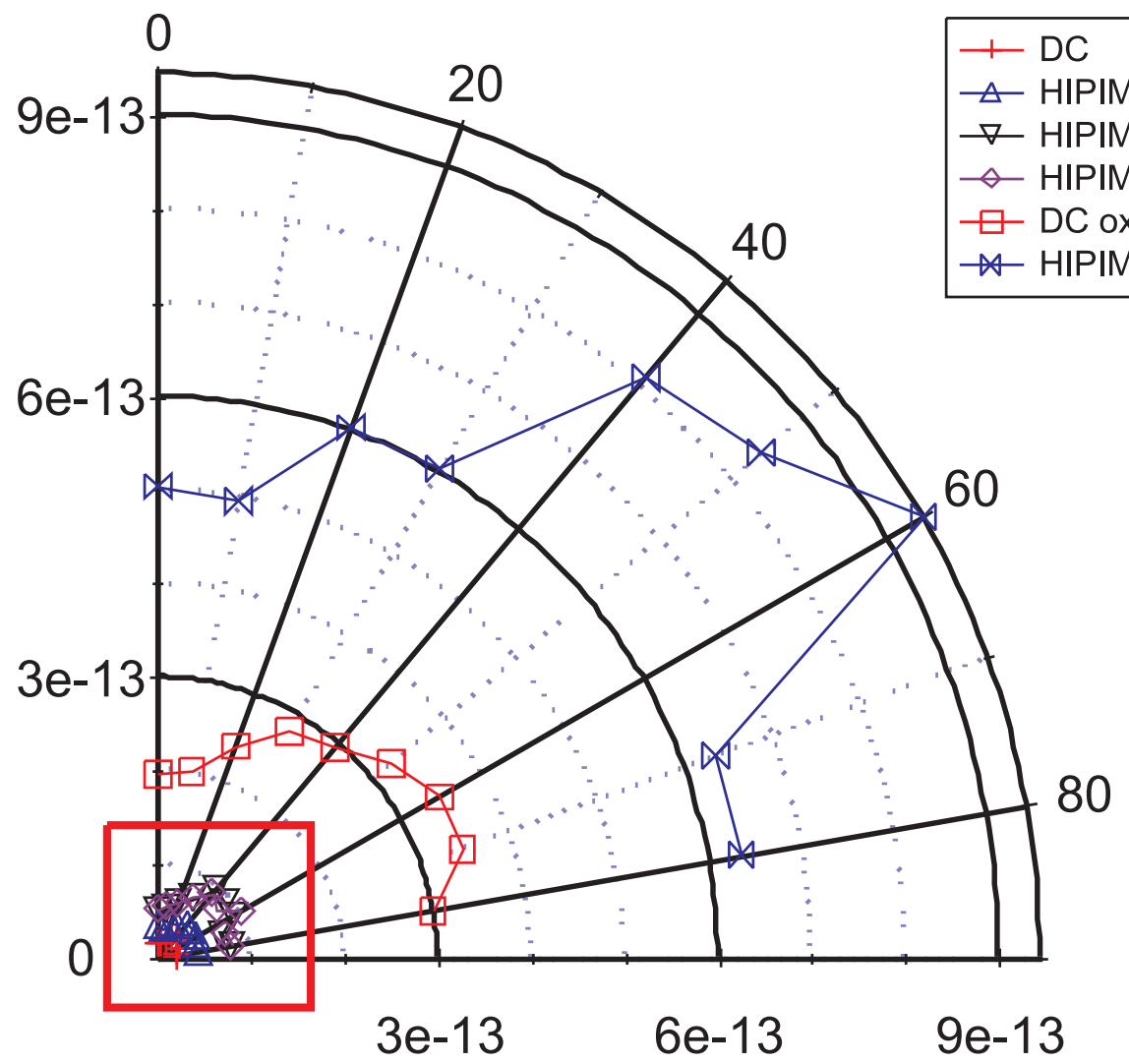

E-flux/Ti-particle (mW s)

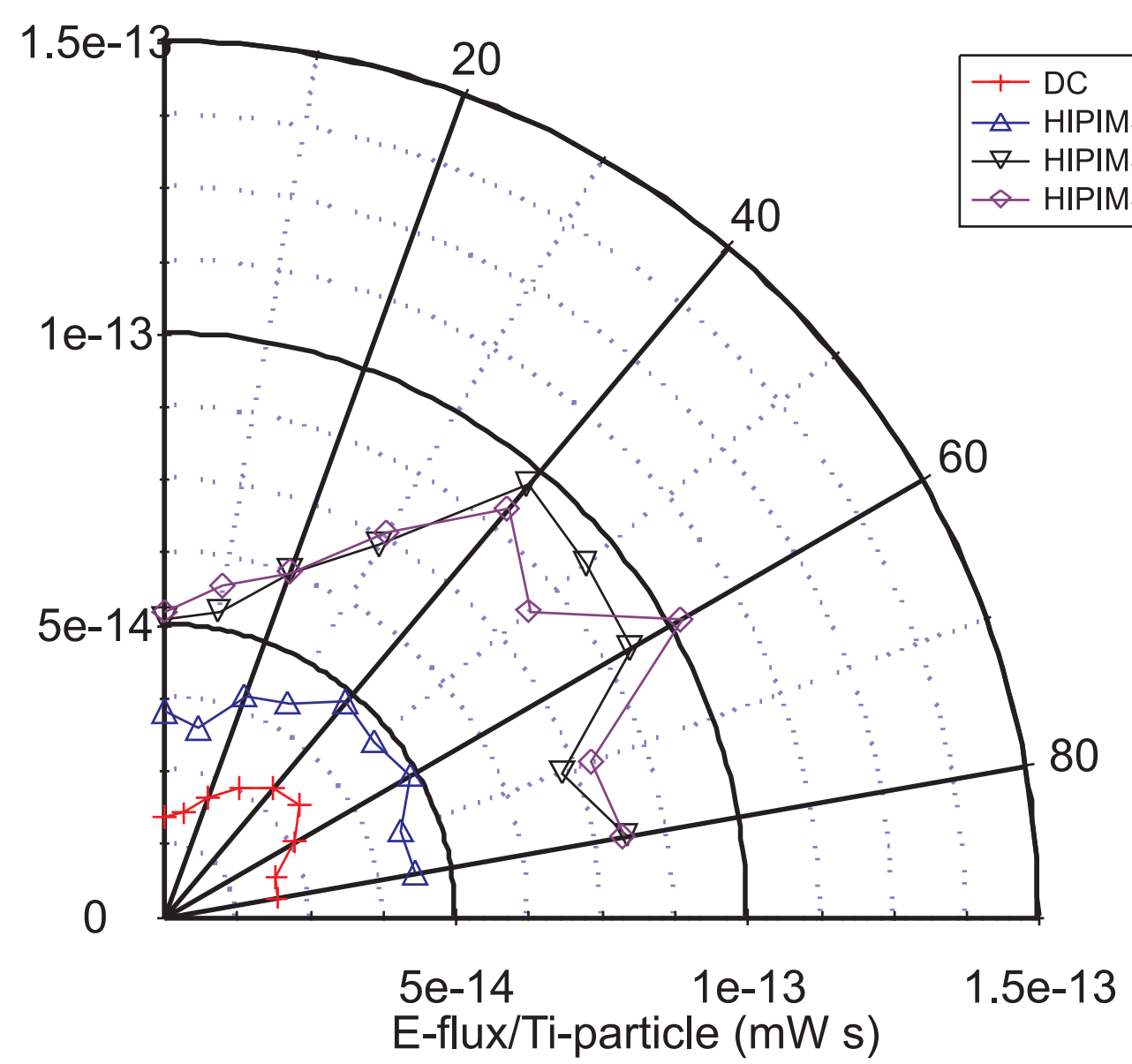

Figure 5 (WouterLeroy_Revision_Figure5.eps) 